\title{
Using the Morris sensitivity analysis method to assess the importance of input variables on time-reversal imaging of seismic sources
}

\author{
Anna Franczyk ${ }^{1}$ (D) \\ Received: 8 November 2018 / Revised: 20 August 2019 / Accepted: 3 September 2019 / Published online: 13 September 2019 \\ (c) The Author(s) 2019
}

\begin{abstract}
The time-reversal imaging method has become a standard technique for seismic source location using both acoustic and elastic wave equations. Although there are many studies on the determination of the relevant parameter for visualization of the time-reversal method, little has been done so far to investigate the accuracy of seismic source location depending on parameters such as the geometry of the seismic network or underestimation of the velocity model. This paper investigates the importance of the accuracy of seismic source location using the time-reversal imaging method of input variables such as seismic network geometry and the assumed geological model. For efficient visualization of seismic wave propagation and interference, peak-to-average power ratio was used. Identification of the importance of variables used in seismic source location was obtained using the Morris elementary effect method, which is a global sensitivity analysis method.
\end{abstract}

Keywords Seismic source location $\cdot$ Time-reversal imaging $\cdot$ Sensitivity analysis

\section{Introduction}

Time-reversal imaging (TRI) utilizes the fact that the wave equation is reversible in time and, under the assumption that energy dissipation is ignored, the observed wave propagation is also symmetrical in relation to the origin time. Emission of the wave from the source towards the sensors runs along a positively oriented time axis. For the negative time direction, the time-reversed recorded signals back-propagate from the sensor positions. The energy is then focused at the area where the source is located. The quality of focusing depends largely on knowledge of the velocity structure of the geological media in which the wave propagates and the geometry of the sensor network. The TRI technique was introduced initially for submarine communication (Parvulescu and Clay 1965); it was publicized and further expanded by Fink (Fink et al. 1989; Fink 1992, 1997). TRI techniques have been used for both seismic location and source mechanism identification (Gajewski and Tessmer 2005; Larmat et al. 2006, 2010; Kawakatsu and Montagner 2008; Steiner and Saenger 2010; Artman et al. 2010; Debski 2015), wave field

Anna Franczyk

franczyk@geol.agh.edu.pl

1 AGH - University of Science and Technology, Kraków, Poland migration (Baysal et al. 1983; McMechan 1983; Tarantola 1988; Fichtner et al. 2006), microseismic location (Wang et al. 2016), and structure imaging in complex geological conditions like salt domes (Willis et al. 2006; Lu 2008). There are also many studies investigating the use of various imaging parameters. Although TRI methods are mostly based on the maximum value, the following were tested as imaging parameters: maximum horizontal and vertical displacement components (Hu and McMechan 1988; Steiner and Saenger 2012; Saenger 2011); maximum particle velocity (Steiner et al. 2008); strain components (Blomgren et al. 2002); maximum amplitude of pressure value (Gajewski and Tessmer 2005); energy density of stress components (Gajewski and Tessmer 2005; Saenger 2011); maximum $P$ wave and $S$ wave energy density, maximum energy density, geometric mean (Nakata and Beroza 2016), and maximum stress components (Saenger 2011). Microseismic events were also refocused using separate $P$ and $S$ waves's backpropagation to the original source location (Douma and Snieder, 2015). In acoustic wave field modelling, TRI techniques based on the maximum absolute pressure value show lower resolution than TRI techniques based on peak-to-average power ratio (Franczyk et al. 2017). PAPR can be also applied to the reconstruction of seismic events that are not separated in time or space, for which using the maximum value of 
pressure as an imaging parameter can be problematic (Anderson et al. 2011).

Application of TRI to the location of seismic sources is an issue that strictly depends on the quality of input data. Both a lack and an excess of information can affect the quality of the location procedure. Moreover, possible perturbations in input variables propagate through the model and affect the results. Perturbations in input data affect the results of TRI location differently and therefore have varying degrees of importance. Identification and quantification of the importance of input variables provides insight into which variables are crucial to the TRI source location procedure and can significantly improve the accuracy of seismic source location. The importance of input variables can be determined with sensitivity analysis.

Sensitivity analysis (SA) is a commonly used technique to identify the relationship between the inputs and outputs of a computational model (Saltelli et al. 2000). There are many studies from various scientific fields on the theory and applications of SA (Frey and Patil (2002), Saltelli et al. (2000, 2004, 2008), Ratto et al. (2007), Jakeman et al. 2006). In this paper, the accuracy of seismic source location in relation to the accuracy of the estimation of the seismic wave speed and the configuration of the receiver network is presented. The evaluation is carried out by implementation of the Morris Screening method (Morris 1991), a reliable and efficient SA technique.

This paper begins with brief outline of the TRI method with the use of the peak-to-average power ratio; this is followed by a short description of the SA method used in computations and its sampling strategy. A description of the use of SA in TRI location is then presented, including discussion of the selection of the system policy variables and their methods of perturbation. Sensitivity analysis results are then described, followed by the conclusion and resulting recommendations.

\section{Time-reversal imaging method with the use of peak-to-average power ratio}

The time-reversal imaging method presented in this paper is based on the two-dimensional acoustic wave field equation described by Eq. 1:

$\frac{\partial^{2} P}{\partial t^{2}}=\kappa\left[\frac{1}{\rho}\left(\frac{\partial^{2}}{\partial x^{2}}+\frac{\partial^{2}}{\partial y^{2}}\right) P+\nabla \cdot f\right]$

where $P(x, y)$ is the pressure field, $f$ denotes body force, $\rho$ $=(x, y)$ denotes the density of the medium and $\kappa=\kappa(x, y)$ is the bulk modulus. Forward simulation was used to produce a signal with full control of its origins. As a result of forward simulation, synthetic seismograms for a given geological model and receiver network were computed. In the next stage of computation, synthetic seismograms were reversed in time in order to perform the role of the source function during backward propagation of the seismic wave. The acoustic wave field equation (Eq. 1) was transformed to a system of first-order hyperbolic linear equations (Virieux 1986) and solved numerically using a staggered-grid finitedifference method.

Boundaries at the grid periphery were coded to satisfy the wave absorbing conditions (Cerjan et al. 1985). The source wavelet in the forward modelling was estimated with the Ricker wavelet. We restricted modelling to nondissipative media for simplicity.

Seismic source location with TRI was performed using the peak-to-average power ratio (PAPR) as an imaging parameter. The PAPR indicates how extreme the peaks are in a waveform; therefore, computation node with the highest value of the PAPR parameter may indicate the location of the seismic source. The PAPR is a positive and dimensionless quantity which can be defined as a ratio of the peak value of a waveform to its RMS value.

The value of the PAPR coefficient can be computed from Eq. 2:

$\operatorname{PAPR}(x)=\frac{\max _{0 \leq t \leq T}|P(x, t)|^{2}}{\frac{1}{T} \int_{0}^{T}|P(x, t)|^{2} \mathrm{~d} t}$,

where $P(x, t)$ is the pressure value at each computational node, $t$ is time index and $T$ is the number of computational time steps in the backward propagation of the seismic wave algorithm.

In the TRI procedure, both the maximum absolute pressure value and the PAPR coefficient can be used. In both cases, the enormous values computed in the given computational node corresponding to the source point location are maintained during the whole process of backward wave propagations. Although both imaging parameters are calculated in much the same manner, the TRI with PAPR coefficient shows a higher spatial resolution that can improve the location of seismic event sequences (Franczyk 2017).

The result of the TRI location procedure with PAPR coefficient for the synthetic example based on Marmousi model (Versteeg 1994) is presented in Fig. 1. Location procedure was conducted using five example receivers' network marked in Fig. 1c-f with red triangles. For all the cases considered, a single seismic source placed at the same point $((x, z)=(3750,1750) \mathrm{m}$, marked in white star in Fig. 1a) was introduced. The results of TRI location with PAPR coefficient for surface sensor network with various receivers and spacing are presented in Fig. 1b-e. The results of location 

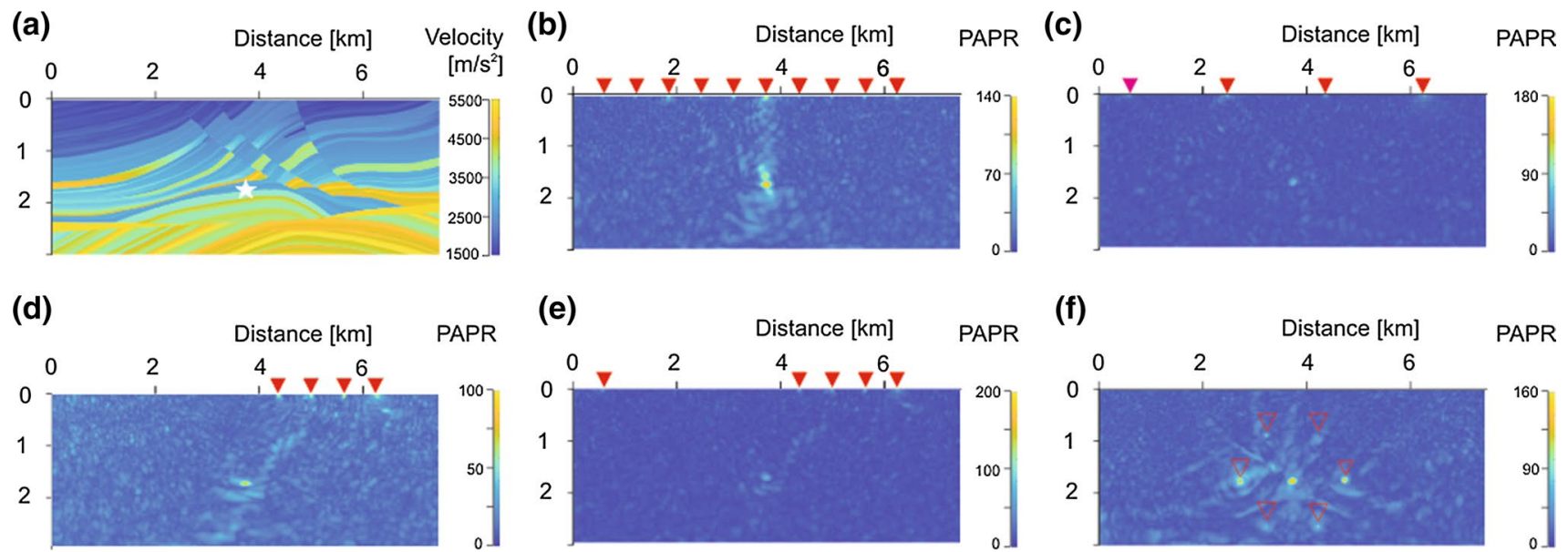

Fig. 1 PAPR distribution (b-f) computed for Marmousi (Martin et al. 2006) velocity model (a) for five example receivers' network configurations. Source point, located in the point: $(x, z)=(3750,1750) \mathrm{m}$, is marked with white star, whereas receivers' network is marked with red triangles $(\mathbf{b}-\mathbf{f})$. PAPR distribution computed for surface network with various receivers and spacing are presented in subplot (b-e). PAPR distribution computed for network surrounding source is presented in the subplot (f)

sensitivity analysis was conducted. To make the conclusions independent of a particular geological model, the sensitivity analysis was performed in the homogenous model. The issue of underestimating of the velocity model has also been considered in sensitivity analysis of the TRI location procedure.

\section{Morris screening}

The Morris method (Morris 1991) is a specialized randomized one-at-a-time (OAT) method that is considered to be a global method of SA. In OAT SA design, all input variables in question are changed by the same relative amount. The Morris method takes into account changing the variable in question between a pair of model simulations; this distinguishes it from the traditional approach of OAT analysis. Identification and ranking of the important variables are done on the basis of the difference computed between a pair of model simulations (Morris 1991; Campolongo et al. 2000).

All input values of the variables in question are selected according to a predetermined algorithm. The algorithm starts at a randomly chosen point in the k-dimensional space (Fig. 2) and creates a trajectory through all the $k$-dimensional variable space. The trajectory is built with $k+1$ points. Two adjacent points differ by standardized step $\Delta_{i}$ only in one dimension of the $k$-dimensional variable space. The coordinates of every point of the single trajectory are used as input values to the computational algorithm. Step-by-step construction of the single trajectory for $k=3$ parameters is presented in Fig. 2.

In the construction of the single trajectory, it is assumed that each input factor can take a discrete number of values, network geometry on accuracy of seismic source location, 

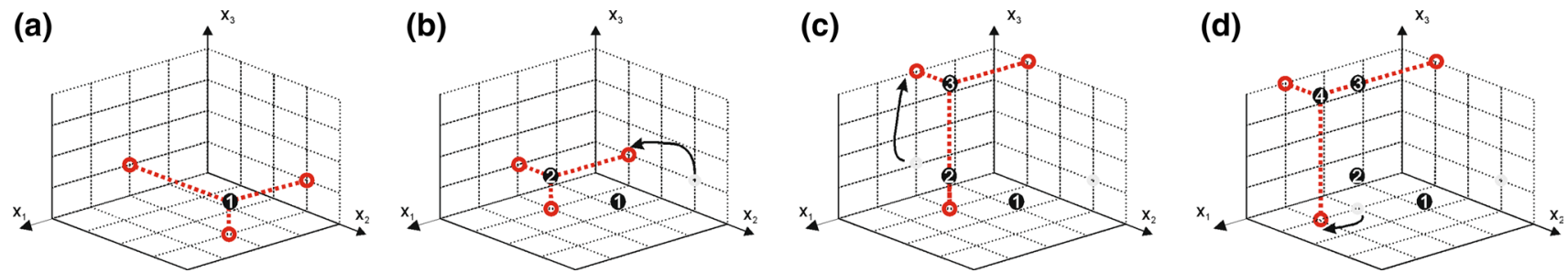

Fig. 2 An example of a single trajectory constructed for the three-dimensional space of input parameters. The trajectory is built with four points: one randomly chosen (a) and three points created as a result of changing one value at each step (b-d)

called levels, which are chosen within the factor range of variation. The levels, denoted as $p$, sampled each factor range evenly: $X_{\mathrm{i}}=\{0,1 /(p-1), 2 /(p-1), \ldots(p-2) /$ $(p-1), 1\}$. The value for $p$ typical used in SA are 4, 6 and 8 , which corresponds to the 25 th, 17 th and 12.5 th percentile of the uniform distribution of the input (Morris 1991; Campolongo et al. 2007). The $k$-dimensional space is thus transformed in $k$-dimensional $p$-level grid with the magnitude of the experiment step $\Delta$ equal to multiple of $1 /(p-1)$. Figure 2 shows $k=3$ variable model for which $p=5$ levels were assumed $X_{\mathrm{i}}=\{0,1 / 4,2 / 4,3 / 4,1\}$ with experiment step of $\Delta=1 / 4$. Base value of $\quad X^{*}=\{1 / 2,3 / 4,1 / 4\}$ has been randomly selected (Fig. 2a). The final trajectory matrix $B^{*}$ is computed as (Morris 1991):

$B *=\left(J_{k+1,1} X *+\frac{\Delta}{2}\left[\left(2 B-J_{k+1, k}\right) D *+J_{k+1, k}\right]\right) P *$

where $J_{k+1, k}$ and $\mathrm{J}_{k+1, l}$ are $k+1$-by- $k$ and $k+1$-by- 1 dimensional matrix and vector of 1 's, $B$ is $(k+1, k)$-dimensional sampling matrix containing only 0 's and 1 's arranged in the lower left triangle unit matrix; $D^{*}$ is $k$-dimensional diagonal matrix in which each element is either 1 or -1 , with equal probability, and $P^{*}$ is a $k$-by- $k$ random permutation matrix in which each column contains one element equal to 1 , and all others equal to 0 , and no two columns have 1's in the same position.

To implement the Morris Screening method, a number, $r$, of different trajectories through variable space have to be constructed. The choice of $p$ is strictly linked to the choice of $r$. When the number of trajectories $r$ is small, it is possible that not all the possible factor levels are explored. Also taking too many levels (assuming high value of $p$ ) when it is not coupled with high value of $r$ may waste the experimental results as many possible levels will remain unexplored. It is assumed that valuable results can be obtained for $p=4$ and rin the range 4-10 (Saltelli et al. 2004).

The sensitivity test is based on the elementary effect $\left(\mathrm{EE}_{i, j,} i=1, \ldots r, j=1, \ldots k\right)$, which is separately computed for each trajectory and each variable of interest. $\mathrm{EE}_{\mathrm{i}, \mathrm{j}}$ is calculated based on the output values obtained for two adjacent points of the trajectory. The elementary effect can be described as:
$\mathrm{EE}_{i, j}=\frac{Y\left(X_{1}, X_{2}, \ldots, X_{i}+\Delta_{i}, \ldots \ldots, X_{k},\right)-Y\left(X_{1}, X_{2}, \ldots, X_{i}, \ldots \ldots, X_{k}\right)}{\Delta_{i}}$

All $\mathrm{EE}_{i, j}$ values computed for randomly chosen trajectories are used to compute final sensitivity measures such as:

mean of absolute value of the elementary effect of the $j$-th parameter (Campolongo et al. 2000)

$\mu_{j}^{*}=\frac{1}{r} \sum_{i=1}^{r}\left|\mathrm{EE}_{i, j}\right|$

standard deviation of the elementary effect

$\sigma_{j}=\sqrt{\frac{1}{r} \sum_{i=1}^{r}\left(\mathrm{EE}_{i, j}-\frac{1}{r} \sum_{i=1}^{r}\left(\mathrm{EE}_{i, j}\right)\right)^{2}}$

On the basis of the values $\mu_{j}$ and $\sigma_{j}$, all input parameters are classified in three groups: inputs with negligible effects; inputs with large linear effects without interactions; and inputs with large nonlinear or interaction effects.

\section{Numerical experiment}

The sensitivity analysis was performed using synthetic tests. Tests were carried out for the established geological model that was known in advance and the known location of the seismic wave sources. This allowed comparison of the TRI location results depending on different configurations of the receiver network geometry and underestimation of the velocity model. The accuracy of the location procedure was assumed here as the difference between the known location of the seismic source and the location corresponding to the highest PAPR value. The results presented in this work were obtained for a homogenous model with constant velocity. For the numerical tests, one source located in the middle of the computational model was established. The Ricker signal was used as a source function in the forward modelling. The synthetic seismograms computed in the forward modelling 


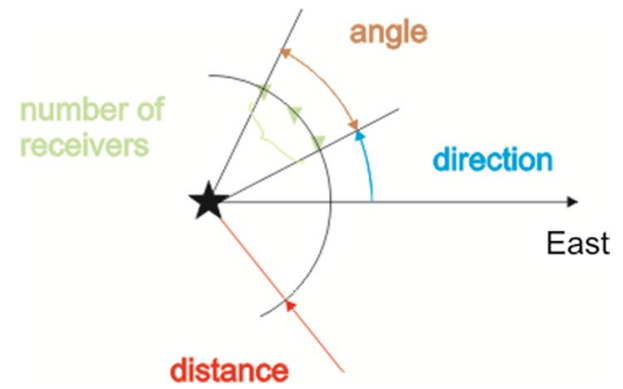

Fig. 3 Geometry of the receiver network and its parametrization by number of sensors in the receiver network, distance from source point to receiver point, direction (location of the first sensor in the network) and angle (spatial location of the sensors around the source)

Table 1 The range of variability of receiver network geometry parameters (input values)

\begin{tabular}{lll}
\hline Parameter & Min & Max \\
\hline Number of receivers & 4 & 10 \\
Angle & $\pi / 4$ & $\pi 2$ \\
Distance from source to receiver points & 50 & 500 \\
$\quad[\mathrm{~m}]$ & & \\
Direction & 0 & $\pi 2$ \\
\hline
\end{tabular}

were reversed and used as an input for the backward modelling stage of the TRI location procedure. Four parameters were used in order to describe the seismic network geometry: the number of receiver network sensors, the angle to fill, the distance between the source and receivers, and the direction of the receiver network, defined as the angle between the line passing through the first geophone and the wave source and the geographical eastern axis, anticlockwise (Fig. 3).

As assumed in the sensitivity analysis, the ranges over which individual parameters of the TRI location procedure can vary are summarized in Table 1.

The variability of receiver network parameters was determined both to ensure the minimum number of receivers needed to locate the seismic event used in the traditional location algorithm and to allow highly sophisticated interpretation (Dresen and Ruter 2013). The variability of the angle to fill parameter reflects the distribution of geophones in one direction and the perfect full coverage of the receiver network. The source point-receiver distance was limited by the size of the assumed geological model. The results of the TRI-based location procedure that utilizes acoustic modellings in a homogenous geological model do not depend on the direction from which the seismic signal arrives. Therefore, in this case the parameter describing the direction between the source point and the sensor network can be interpreted as the sensitivity of the location procedure itself. In addition to the 4 parameters described above, the effect of the velocity model underestimating the TRI location results was additionally examined. The results presented in this article are based on the assumption that the underestimation of the velocity model may be as much as $10 \%$ of its actual value.

In this study, the Morris sampling was designed to calculate 60 elementary effects for each input. Although many applications of the Morris method use a low repetition number, e.g., $r=10-20$ (Campolongo et al. 2007), it has been shown that $r$ has a significant effect on the identified parameter sensitivity (Cropp and Braddock 2002). As a result of the assumed number or trajectories, $360\left(=r^{*}(k+1), k=5\right)$ TRI location procedures were launched. For each simulation, modelling of seismic wave propagation was performed twice: in the forward modelling stage to calculate synthetic seismograms and in the backward modelling stage, in which the calculated seismograms were used as a source signal. An example trajectory of the input values, obtained as a result of Morris sampling, is presented in Fig. 4.

\section{Results and discussion}

The Morris results are evaluated by comparing the mean and standard deviation of the distribution function of each input (Fig. 5). The mean of the absolute value of the elementary effect (Eq. 5) provides the overall sensitivity of the $i$ th input variable on the output response; in this case, this is the accuracy of the TRI location procedure. The higher the calculated value of $\mu^{i}$, the more sensitive the location procedure is to the $i$ th input variable. A standard deviation of elementary effect (Eq. 6) indicates possible interactions with other variables and/or that the variable has a nonlinear effect on the output (Campolongo and Braddock 1999).

In Fig. 5, a graph linking sensitivity measurements of mean value $\mu_{i}{ }^{*}$ and standard deviation $\mu_{i}$ of all input parameters is presented.

The plot of the mean value and standard deviation $\left(\mu_{i}{ }^{*}\right.$, $\sigma_{i}$ ) pair suggests that the most influential parameter of the TRI location procedure is the source-receiver distance. The other four parameters are much less important for the accuracy of seismic source location: the spatial spread of sensors (angle to fill), the number of receivers, the direction between the source point and the receiver network, and underestimation of the velocity model. Moreover parameters related to the geometry of the sensor network indicate a nonlinear effect or interaction with other variables because mean value $\mu_{i}^{*}$, and standard deviation have the same order of magnitude for the direction, number, and angle parameters. In this case, direction indicates the quality of the location procedure because the results of the location procedure based on TRI with acoustic modellings are insensitive to the direction 

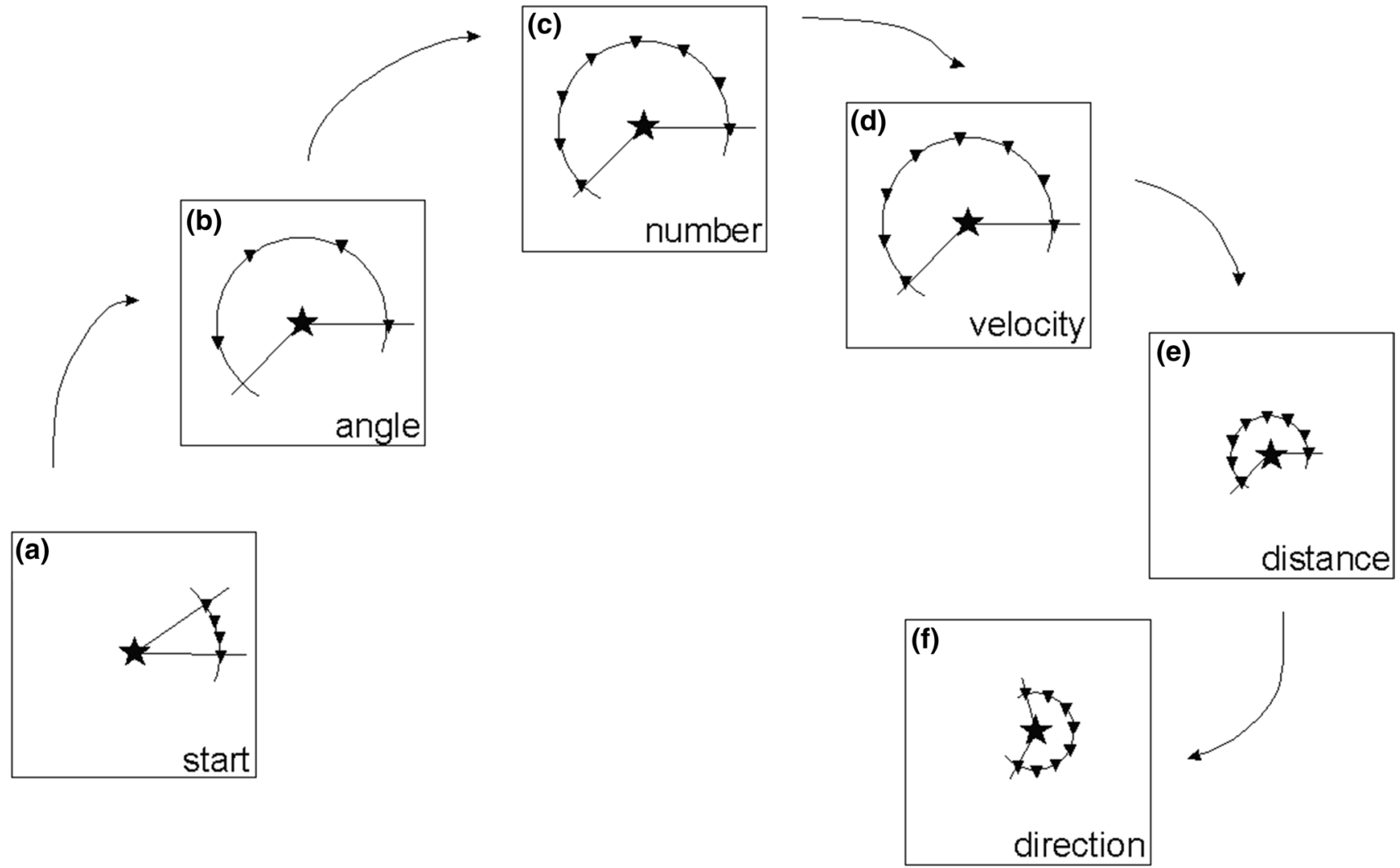

Fig. 4 Single-input value trajectory constructed by changing the value of one input parameter at a time. Trajectory was initiated with the randomly chosen values of the input parameters (a). Then trajec-

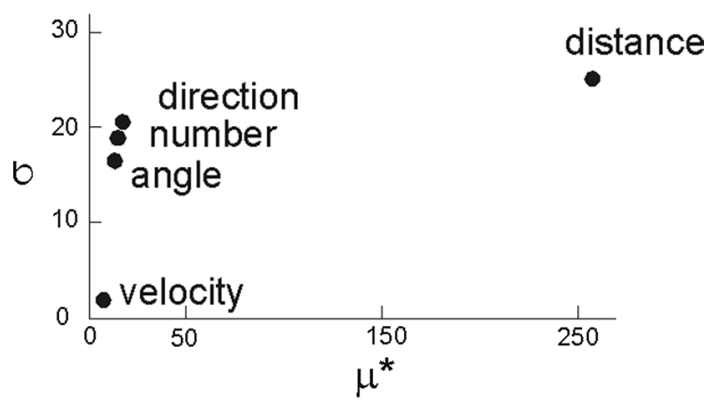

Fig. 5 Mean and standard deviation of the distribution function of each input. Each circle corresponds to one input of the model

between the source point and the location of the seismic network.

Most location errors were related to increased PAPR parameter values observed around the sensor network location. This was due to the interference of the seismic signal propagating from neighbouring receivers in the backpropagation stage. In order to improve the quality of the seismic source location, some improvements to tory was built through the successive change of the value of only one factor at a time (b-f). The TRI procedure was carried out for all six sets of the input parameters values

the location algorithm were introduced. As before, the improved algorithm was based on the maximum value of the PAPR; however, some restrictions of which areas could be searched were added. Restricted areas excluded from the searching algorithm were located around the sensor network. They were constructed using PAPR values calculated in the known coordinates of sensors. The threshold value designating the area of exclusion was computed using the equation:

$\mathrm{Th}_{i}=k \cdot \frac{\mathrm{PAPR}_{i}}{P A P R_{\text {max }}}$

where $\mathrm{Th}_{i}$ is the threshold value of the $i$ th sensor, $\mathrm{PAPR}_{i}$ is the PAPR value calculated in the known $i$ th receiver location, $\mathrm{PAPR}_{\max }$ is the maximum value of the PAPR coefficient obtained for the whole computational model, $k$ is a coefficient (in this case 0.85 ).

Two examples of TRI location procedures without restricted regions and with restricted regions determined for $k=0.95$ and $k=0.85$ are presented in Fig. 6 . A coefficient value equal to 0.85 (determined as a result of numerical experiments) was applied for the improved 


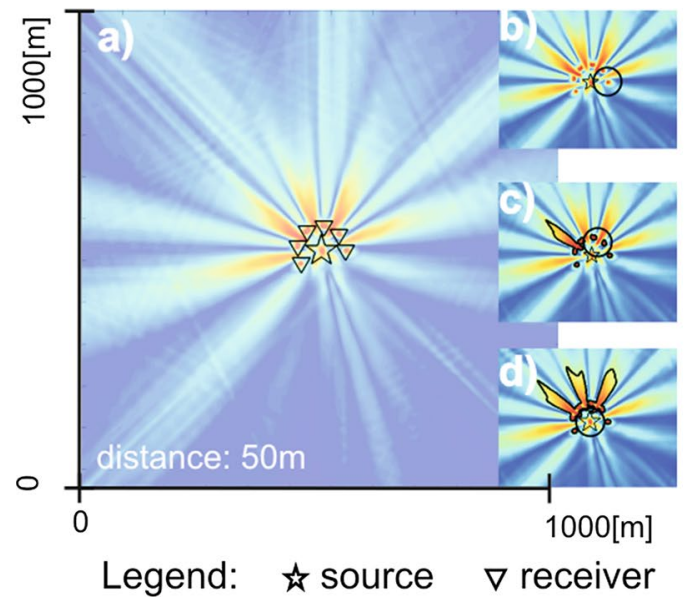

Fig. 6 Two examples of the TRI location algorithm. Pictures a and e show results of TRI procedure with the known a priori seismic source and receivers' location. Results of the TRI location procedure without

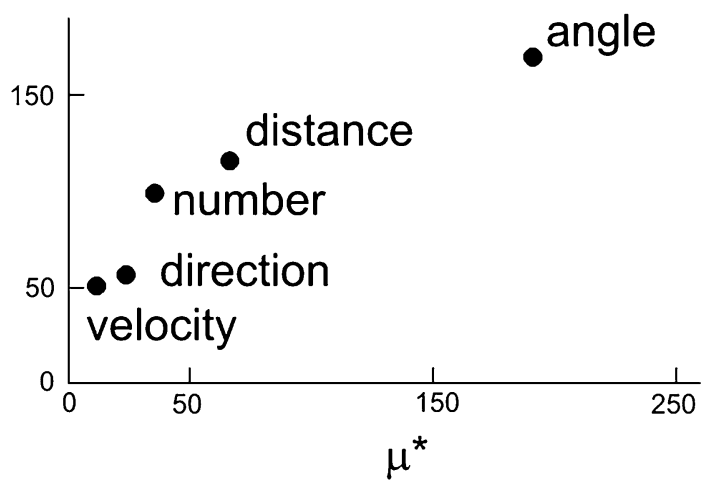

Fig. 7 Mean and standard deviation of the distribution function of each input computer for the TRI location with restricted area and a coefficient $k$ of 0.85

TRI location procedure. In most cases, it resulted in the improvement of the location procedure (Fig. 6d). However, for a receiver network with a small distance between sensors, the use of restricted areas in the location procedure significantly increased the location error (Fig. 6h).

Figure 7 presents a graph linking sensitivity measurements $\mu_{i}^{*}$ and $\sigma_{i}$ of all input parameters of the improved location procedure.

The plot of the $\left(\mu_{i}{ }^{*},{ }_{i}\right)$ pair calculated for the improved location procedure suggests that the parameters that most influence the accuracy of TRI location are those related to the sensor network geometry. The results of sensitivity analysis indicate that the spatial location of the sensors around the source (described as an angle in Fig. 7) is the most influential factor. The TRI location algorithm is also strongly dependent on such seismic network geometry parameters as the number of sensors (the number of

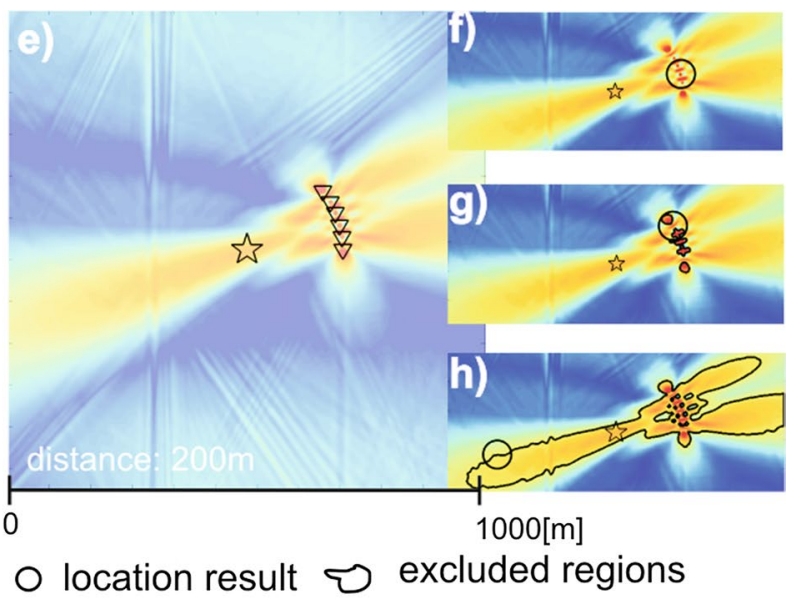

a restriction area (b and $\mathbf{f})$ and with the excluded area computed for $k$ $=0.95$ (c and $\mathbf{g}$ ) and $k=0.85(\mathbf{d}$ and $\mathbf{h})$ are marked with the centre of the circle

seismograms used in TRI) and the source-receiver distance. Sensitivity analysis shows not only a significant value of means, but also large values of the standard deviation of the TRI location results. Analysis of the location results carried out for different network geometries showed that both an increase and a decrease in the number of receivers and the source-receiver distance can introduce location errors. This is shown in the plot of $\left(\mu_{i}^{*}, \sigma_{i}\right)$ in large values of mean and standard deviation of both parameters. Large values of standard deviation computed for all parameters related to the sensor network indicate their nonlinear influence on location results. Moreover, standard deviation values that are higher than the mean value calculated for the parameters of the source-receiver distance and the number of sensors may indicate interaction between these two parameters. Sensitivity analysis showed that velocity underestimation was the least sensitive parameter of the TRI location algorithm. Such a low ranking is associated with the simplified velocity model adopted in the calculations. This surprisingly low ranking of velocity underestimation may also be explained by the location procedure itself. The results of the sensitivity analysis indicate that the location procedure (described as direction in Fig. 7) has a much greater impact on the accuracy of TRI location than velocity underestimation. Strong side lobes and inappropriate estimation of excluded regions introduced variance of location procedure. It is especially visible in the position of "direction" parameters on Figs. 6 and 7. The application of the excluded regions (with $k=0.85$ ) improves the quality of location procedure because the influence of the "direction" parameter has been marginalized compared to its position presented in Fig. 6. Moreover, the statistical approach used in the 
sensitivity analysis, based on the assumption large number of trajectories, can give credibility to its results.

\section{Conclusion}

Seismic source location using TRI techniques is an example of a computational problem whose accuracy depends on many factors. Proper selection and fine-tuning of location procedure parameters becomes challenging for effective application of imaging techniques in seismic source location. Hence, the Morris screening approach was applied to help determine the importance of these parameters in relation to the accuracy of the location procedure. An iterative procedure was executed in order to find out the optimal restricted area for the improved TRI location procedure. The selection of the best algorithm was also based on sensitivity analysis. The use of an acoustic wave equation in the TRI location procedure makes it possible to determine the best location procedure as that which has the least sensitivity to the direction of the seismic propagation.

The sensitivity analysis is able to indicate the parameters that are primarily responsible for the variance in the output values. The significant parameters are related to the geometry of the sensor network. This information helps understand what causes the uncertainty and, hence, how it can be remedied. The results presented in the work particularly indicate that selection of the correct number and the optimal distribution of sensors (the records of which are used in the TRI algorithm) are a key aspect of location accuracy.

The results presented in this work can also help in determining the origin time of the seismic source. Refining the spatial location by tuning the optimal configuration of the sensor network will allow areas of potential seismic wave locations to be determined. With better location of seismic sources, we can go back through the time snapshots to examine the time at which the amplitude achieved its maximum value, thus indicating the origin time of the seismic event. This makes it possible to determine the time history of the source emissions and the time sequence for both single and multiple sources.

The results of the sensitivity analysis presented in this work showed little effect of the underestimation of the velocity model on the accuracy of the TRI location procedure. These results are due to the simplified homogeneous velocity model assumed in the numerical experiment. For a location procedure performed for real data, underestimation of the velocity model would have a much greater impact on the accuracy of the TRI location procedure. The impact of velocity underestimation for more complicated geological models on the accuracy of the TRI location procedure is currently under investigation. The results of the sensitivity analysis bring us closer to applying the TRI location method to real data.

Acknowledgements This work was supported in part by the AGH University of Science and Technology, Faculty of Geology, Geophysics and Environmental Protection under statutory Project 11.11.140.613 and National Science Centre, Poland under Grant No. 2015/17/B/ ST10/01946.

Open Access This article is distributed under the terms of the Creative Commons Attribution 4.0 International License (http://creativeco mmons.org/licenses/by/4.0/), which permits unrestricted use, distribution, and reproduction in any medium, provided you give appropriate credit to the original author(s) and the source, provide a link to the Creative Commons license, and indicate if changes were made.

\section{References}

Anderson BE, Griffa M, Ulrich TJ, Johnson PA (2011) Time reversal reconstruction of finite sized sources in elastic media. J Acoust Soc Am 130(4):EL219-EL225.

Artman B, Podladtchikov I, Witten B (2010) Source location using time-reverse imaging. Geophys Prospect 58(5):861-873. https:// doi.org/10.1111/j.1365-2478.2010.00911

Baysal E, Kosloff D, Sherwood JWC (1983) Reverse time migration. Geophysics 48(11):1514-1524. https://doi.org/10.1190/1.1441434

Blomgren P, Papanicolaou G, Zhao H (2002) Super-resolution in timereversal acoustics. J Acoust Soc Am 111(1):230-248. https://doi. org/10.1121/1.1421342

Campolongo F, Braddock RD (1999) The use of graph theory in the sensitivity analysis of the model output: a second order screening method. Rel Eng Syst Saf 64(1):1-12

Campolongo F, Cariboni J, Saltelli A (2007) An effective screening design for sensitivity analysis of large models. Environ Modell Softw 22:1509-1518

Campolongo F, Kleijnen J, Andres T (2000) Screening methods. In: Saltelli A, Chan K, Scott EM (eds) Sensitivity analysis. Wiley, Chichester, pp 65-80

Cerjan C, Kosloff D, Kosloff R, Reshef M (1985) A non-reflecting boundary condition for discrete acoustic and elastic wave equations. Geophysics 50(4):705-708. https://doi.org/10.1190/1.14419 45

Cropp R, Braddock R (2002) The new Morris method: an efficient second-order screening method. Reliab Eng Syst Safe 78(1):77-83

Debski W (2015) Using meta-information of a posteriori Bayesian solutions of the hypocentre location task for improving accuracy of location error estimation. Geophys J Int 201(3):1399-1408. https://doi.org/10.1093/gji/ggv083

Douma J, Snieder R (2015) Focusing of elastic waves for microseismic imaging. Geophys J Int 200(1):390-401

Dresen L, Ruter H (2013) Seismic coal exploration: in-stream seismic. Elsevier, Amsterdam

Fichtner A, Bunge H-P, Igel H (2006) The adjoint method in seismology I. Theory Phys Earth Planet Int 157(1-2):86-104. https://doi. org/10.1016/j.pepi.2006.03.016

Fink M (1992) Time reversal of ultrasonic field—part I: basic principles. IEEE Trans Ultrason Ferroelectr Freq Control 39(5):555566. https://doi.org/10.1109/58.156174

Fink M (1997) Time reversed acoustics. Phys Today 50(3):34-40. https ://doi.org/10.1063/1.881692

Fink M, Prada C, Wu F, Cassereau D (1989) Self-focusing in inhomogeneous media with time reversal acoustic mirrors. IEEE 
Ultras Symp Proc 1(2):681-686. https://doi.org/10.1109/ULTSY M.1989.67072

Franczyk A, Leśniak A, Gwiżdż D (2017) Acta Geophys 65:299. https ://doi.org/10.1007/s11600-017-0022-0

Frey HC, Patil SR (2002) Identification and review of sensitivity analysis methods. Risk Anal 22(3):553-578

Gajewski D, Tessmer E (2005) Reverse modelling for seismic event characterization. Geophys J Int 163(1):276-284. https://doi. org/10.1111/j.1365-246X.2005.02732.x

Hu LZ, McMechan GA (1988) Elastic finite difference modelling and imaging for earthquake sources. Geophys J Int 95(2):303-313. https://doi.org/10.1111/j.1365-246X.1988.tb00469.x

Jakeman AJ, Letcher RA, Norton JP (2006) Ten iterative steps in development and evaluation of environmental models. Environ Modell Soft 21:602-614

Kawakatsu H, Montagner J-P (2008) Time-reversal seismic source imaging and moment-tensor inversion. Geophys J Int 175(2):686688. https://doi.org/10.1111/j.1365-246X.2008.03926.x

Larmat C, Guyer RA, Johnson PA (2010) Time-reversal methods in geophysics. Phys Today 63(8):31-35. https://doi. org/10.1063/1.3480073

Larmat C, Montagner J-P, Fink M, Capdeville Y, Tourin A, Clévédé E (2006) Time-reversal imaging of seismic sources and application to the great Sumatra earthquake. Geophys Res Lett 33(19):L19312. https://doi.org/10.1029/2006GL026336

Lu R (2008) Time reversed acoustics and applications to earthquake location and Salt Dome Flank imaging, Ph.D. thesis at Massachusetts Institute of Technology

Martin KJ, Wiley R, Marfurt KJ (2006) Marmoousi2: an elastic upgrade for Marmousi. Lead Edge 25(2):156-166. https://doi. org/10.1190/1.2172306

McMechan G (1983) Migration by extrapolation of time-dependent boundary values. Geophys Prospect 31(3):413-420. https://doi. org/10.1111/j.1365-2478.1983.tb01060.x

Morris MD (1991) Factorial sampling plans for preliminary computational experiments. Technometrics 33:161-174

Nakata N, Beroza GC (2016) Reverse time migration for microseismic sources using the geometric mean as an imaging condition. Geophysics 81(2):KS51-KS60

Parvulescu A, Clay CS (1965) Reproducibility of signal transmission in the ocean. Radio Electron Eng 29(4):223-228. https://doi. org/10.1049/ree.1965.0047
Ratto M, Young PC, Romanowicz R, Pappenberger F, Saltelli A, Pagano A (2007) Uncertainty, sensitivity analysis and the role of data based mechanistic modeling in hydrology. Hyrdol Earth Syst Sci 11(4):1249-1266

Saenger EH (2011) Time reverse characterization of sources in heterogeneous media. NDT E Int 44(8):751-759. https://doi. org/10.1016/j.ndteint.2011.07.011

Saltelli A, Chan K, Scott EM (eds) (2000) Sensitivity analysis. Wiley, Chichester

Saltelli A, Ratto M, Andres T, Campolongo F, Cariboni J, Gatelli D, Saisana M, Tarantola S (2008) Global sensitivity analysis: the primer. Wiley, Chichester

Saltelli A, Tarantola S, Campolongo F, Ratto M (2004) Sensitivity analysis in practice. Wiley, New York

Steiner B, Saenger EH (2010) Comparison of 2D and 3D time reverse modelling for tremor source localization. SEG Tech Program Expand Abstr 2010:2171-2175

Steiner B, Saenger EH (2012) Comparison of 2D and 3D time-reverse imaging-a numerical case study. Comp Geosci 46:174-182. https://doi.org/10.1016/j.cageo.2011.12.005

Steiner B, Saenger EH, Schmalholz SM (2008) Time reverse modelling of low-frequency microtremors: application to hydrocarbon reservoir localization. Geophys Res Lett 35(3):L03307. https:// doi.org/10.1029/2007GL032097

Tarantola A (1988) Theoretical background for the inversion of seismic waveforms, including elasticity and attenuation. Pure Appl Geophys 128(1):365-399. https://doi.org/10.1007/BF01772605

Virieux J (1986) P-SV wave propagation in heterogeneous media: velocity-stress finite-difference method. Geophysics 51(4):889 901. https://doi.org/10.1190/1.1442147

Willis ME, Lu R, Burns DR, Toksoz MN, Campman X, de Hoop M (2006) A novel application of time reversed acoustics: salt dome flank imaging using walk away VSP surveys. Geophysics 71(2):A7-A11. https://doi.org/10.1190/1.2187711

Versteeg R (1994) The Marmousi experience: velocity model determination on a synthetic complex data set. Lead Edge 13:927-936. https://doi.org/10.1190/1.1437051

Wang H, Li M, Shang X (2016) Current developments on micro-seismic data processing. J Nat Gas Sci Eng 32:521-537. https://doi. org/10.1016/j.jngse.2016.02.058 